MATEC Web of Conferences 24,03002 (2015)

DOI: $10.1051 /$ matecconf/ 20152403002

(C) Owned by the authors, published by EDP Sciences, 2015

\title{
Bayesian Methods for Nonlinear System Identification of Civil Structures
}

\author{
Joel P. Conte ${ }^{1, a}$, Rodrigo Astroza ${ }^{1,2}$ and Hamed Ebrahimian ${ }^{1}$ \\ ${ }^{1}$ University of California, San Diego, Department of Structural Engineering, La Jolla, CA, USA \\ ${ }^{2}$ Universidad de los Andes, Facultad de Ingeniería y Ciencias Aplicadas, Santiago, Chile
}

\begin{abstract}
This paper presents a new framework for the identification of mechanics-based nonlinear finite element (FE) models of civil structures using Bayesian methods. In this approach, recursive Bayesian estimation methods are utilized to update an advanced nonlinear FE model of the structure using the input-output dynamic data recorded during an earthquake event. Capable of capturing the complex damage mechanisms and failure modes of the structural system, the updated nonlinear FE model can be used to evaluate the state of health of the structure after a damage-inducing event. To update the unknown time-invariant parameters of the FE model, three alternative stochastic filtering methods are used: the extended Kalman filter (EKF), the unscented Kalman filter (UKF), and the iterated extended Kalman filter (IEKF). For those estimation methods that require the computation of structural FE response sensitivities with respect to the unknown modeling parameters (EKF and IEKF), the accurate and computationally efficient direct differentiation method (DDM) is used. A three-dimensional five-story two-by-one bay reinforced concrete (RC) frame is used to illustrate the performance of the framework and compare the performance of the different filters in terms of convergence, accuracy, and robustness. Excellent estimation results are obtained with the UKF, EKF, and IEKF. Because of the analytical linearization used in the EKF and IEKF, abrupt and large jumps in the estimates of the modeling parameters are observed when using these filters. The UKF slightly outperforms the EKF and IEKF.
\end{abstract}

\section{Introduction}

Linear finite element (FE) model updating is one of the most popular approaches for damage identification (DID) of civil structures. In this technique, linear FE models are calibrated using data recorded before and after a damaging event and damage is characterized as the reduction of effective stiffness over one or more regions of the structure. Usually, the linear FE model updating problem is solved using a deterministic approach and the updating process is formulated as a constrained optimization problem. The objective of the optimization problem is to minimize the discrepancy between the simulated and recorded responses from low-amplitude vibrations recorded before and after the structure has experienced damage $[1,2]$. The data may consist of time or frequency domain responses or quantities derived therefrom (e.g., modal parameters). Despite its popularity in the field of structural engineering, linear FE model updating cannot provide any information about inelastic deformations, loss of strength and loss of ductility capacity, which are required for a comprehensive condition assessment of the structure.

Recent years have witnessed important progress in the field of nonlinear FE model updating of civil structures to overcome this limitation [3-5]. These studies have utilized simplified nonlinear structural models with lumped nonlinearities defined empirically, through for example the Bouc-Wen model, to describe the hysteretic response behavior of the structure of interest. However, such models are not commonly used in state-of-the-art mechanics-based structural FE modeling because they cannot adequately simulate the actual nonlinear behavior of civil structures. Recently, the problem of updating mechanics-based nonlinear FE models of structures using input-output data recorded during damage-inducing events has been investigated [6-8]. The main benefit of using advanced FE models is that they are able to capture the damage mechanisms to be detected and identified in the structural system and therefore can provide accurate information about presence, location, type, and extent of damage in the structure. The updated mechanics-based nonlinear FE model can be directly used for rapid postevent condition assessment.

This paper describes the use of the Extended Kalman filter (EKF), Iterated Extended Kalman filter (IEKF), and Unscented Kalman filter (UKF) to update mechanicsbased nonlinear FE models and then compares the performance of the filters for a numerically simulated application example. Implementation of the EKF and IEKF requires the FE response sensitivities with respect to the modeling parameters to be estimated. They are computed using the direct differentiation method (DDM), which is an accurate and computationally efficient approach based on the exact (consistent) differentiation of the FE numerical scheme with respect to the modeling

\footnotetext{
${ }^{\mathrm{a}}$ Corresponding author: jpconte@ucsd.edu
} 
parameters. An application example is presented based on data simulated numerically from a realistic nonlinear FE model of a three-dimensional five-story two-by-one bay reinforced concrete (RC) frame building subjected to bidirectional (horizontal) earthquake excitation..

\section{Nonlinear FE modeling}

Over the years, different approaches have been proposed to model and simulate the nonlinear response of frametype structures subjected to earthquake excitation. Global models, structural FE models, and continuum FE models have been developed and used for this purpose [9]. Structural FE models with distributed plasticity have been shown to provide accurate results in predicting structural response and damage mechanisms observed during experimental tests. In addition, their formulation is simple and they are computationally efficient and feasible also for large systems. As a result, FE structural models of this type have been broadly utilized in research and engineering practice to model frame-type structures.

In this type of structural models, material nonlinearity can develop at any numerically monitored cross section (integration point) along the element length, and the element response is obtained through numerical integration of the section response along the element length. Element cross-sections are discretized into longitudinal fibers, the stress-strain behavior of which is simulated using realistic uniaxial material constitutive laws. The nonlinear section response is obtained by integrating the stress-strain response of the fibers over the cross section. This formulation accounts for the interaction between bending moments and axial force at the section level, while the interaction with the shear force is accounted for at the element level through element equilibrium. It is noted that a uniaxial material model depends on a set of physical and/or empirical timeinvariant parameters. Figure 1 shows a schematic representation of the hierarchical discretization in distributed-plasticity structural FE models of RC frametype structures. More details about this structural FE modeling approach can be found in $[9,10]$.
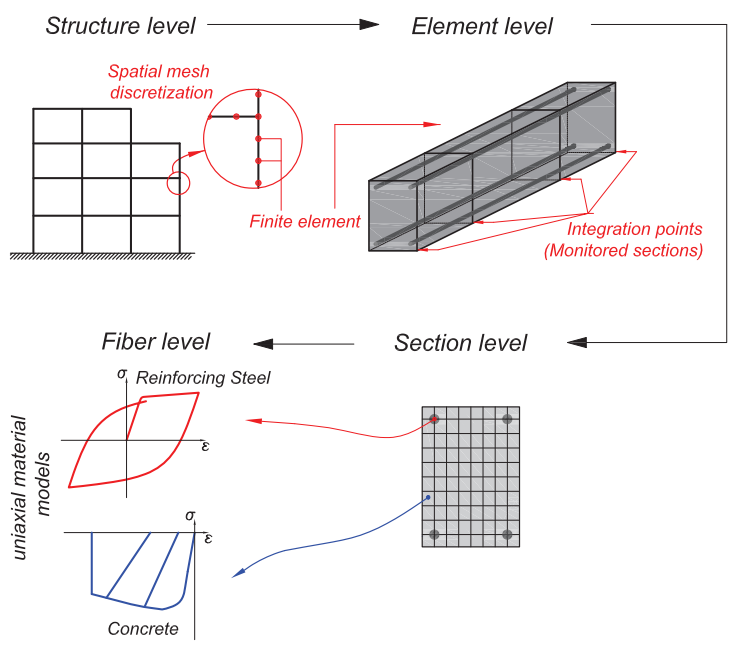

Figure 1. Distributed plasticity FE model of RC frame-type structures.

\section{Problem formulation}

The discrete-time equation of motion of a nonlinear FE model of a structure can be expressed as

$$
\mathbf{M}(\boldsymbol{\theta}) \ddot{\mathbf{q}}_{k+1}(\boldsymbol{\theta})+\mathbf{C}(\boldsymbol{\theta}) \dot{\mathbf{q}}_{k+1}(\boldsymbol{\theta})+\mathbf{r}_{k+1}\left[\mathbf{q}_{k+1}(\boldsymbol{\theta}), \boldsymbol{\theta}\right]=\mathbf{f}_{k+1}(1)
$$

where $\boldsymbol{\theta} \in \mathbb{R}^{n_{\theta}}=$ vector of unknown time-invariant modeling parameters; $\mathbf{q}, \dot{\mathbf{q}}, \ddot{\mathbf{q}} \in \mathbb{R}^{n}=$ nodal displacement, velocity, and acceleration vectors; $\mathbf{M} \in \mathbb{R}^{n \times n}=$ mass matrix; $\mathbf{C} \in \mathbb{R}^{n \times n}=$ damping matrix; $\left.\mathbf{r}(\mathbf{q}(\boldsymbol{\theta}), \boldsymbol{\theta})\right) \in \mathbb{R}^{n}=$ history-dependent internal resisting force vector, $\mathbf{f} \in \mathbb{R}^{n}$ $=$ dynamic load vector, $n=$ number of degrees of freedom, and the subscript $(\ldots)_{k+1}$ indicates the time step. For the case of earthquake uniform base excitation, the dynamic load vector takes the form $\mathbf{f}_{k+1}=-\mathbf{M} \mathbf{L} \ddot{\mathbf{u}}_{k+1}^{g}$, where $\mathbf{L} \in \mathbb{R}^{n \times r}=$ load influence matrix and $\ddot{\mathbf{u}}^{g} \in \mathbb{R}^{r \times 1}=$ input ground acceleration vector with $r=$ number of base excitation components (in the general case of uniform base excitation $r=6$, i.e., 3 translational and 3 rotational base excitation components).

The structural response can be recorded using different types of sensors (e.g., accelerometers, GPS antennas) and at time $t_{k+1}=(k+1) \Delta t$, with $k=0,1, \ldots$ and $\Delta t=$ sampling time step, can be expressed as

$$
\begin{aligned}
& \mathbf{y}_{k+1}=\mathbf{L}_{y}\left[\ddot{\mathbf{q}}_{k+1}^{T}, \dot{\mathbf{q}}_{k+1}^{T}, \mathbf{q}_{k+1}^{T},\left(\ddot{\mathbf{u}}_{k+1}^{g}\right)^{T}\right]^{T}+\mathbf{v}_{k+1} \\
& \mathbf{y}_{k+1}=\hat{\mathbf{y}}_{k+1}+\mathbf{v}_{k+1}
\end{aligned}
$$

where $\mathbf{y} \in \mathbb{R}^{n_{y}}=$ vector of measured response quantities, $\hat{\mathbf{y}} \in \mathbb{R}^{n_{y}}=$ response of the structure predicted from the FE model, $\mathbf{L}_{y} \in \mathbb{R}^{n_{y} \times(3 n+r)}=$ output matrix (known), and $\mathbf{v}_{k+1} \in \mathbb{R}^{n_{y}}=$ simulation error vector (which accounts for measurement error and modeling uncertainty) assumed to be white Gaussian with zero-mean and covariance matrix $\mathbf{R}_{k+1}$, i.e., $\mathbf{v}_{k+1} \sim N\left(\mathbf{0}, \mathbf{R}_{k+1}\right)$. In the present case, effects of modeling uncertainty are not considered and, therefore, $\mathbf{v}_{k+1}$ only represents measurement error.

From Equations (1) and (2), the vector of recorded response quantities at time $t_{k+1}, \mathbf{y}_{k+1}$, can be expressed as a nonlinear function of the modeling parameters $(\theta)$, input ground acceleration time history $\left(\ddot{\mathbf{u}}_{1: k+1}^{g}\right)$, and initial conditions $\left(\mathbf{q}_{0}, \dot{\mathbf{q}}_{0}\right)$ of the FE model, i.e.,

$$
\mathbf{y}_{k+1}=\mathbf{h}_{k+1}\left(\boldsymbol{\theta}, \ddot{\mathbf{u}}_{1: k+1}^{g}, \mathbf{q}_{0}, \dot{\mathbf{q}}_{0}\right)+\mathbf{v}_{k+1}
$$

Here, $\mathbf{h}_{k+1}(\ldots) \mathbf{h}_{k+1}(\cdot)$ is the nonlinear response function of the nonlinear FE model at time $t_{k+1}$ and $\ddot{\mathbf{u}}_{1: k+1}^{g}=$ $\left[\left(\ddot{\mathbf{u}}_{1}^{g}\right)^{T},\left(\ddot{\mathbf{u}}_{2}^{g}\right)^{T}, \ldots,\left(\ddot{\mathbf{u}}_{k+1}^{g}\right)^{T}\right]^{T}$ is the input ground acceleration time history from time $t_{1}$ to $t_{k+1}$. At rest initial conditions are assumed; henceforth $\mathbf{q}_{0}=\dot{\mathbf{q}}_{0}=\mathbf{0}$. 
In the Bayesian parameter estimation framework considered in this paper, the unknown time-invariant modeling parameter vector, $\theta$, is modeled as a random process, the evolution of which is governed by a random walk process. Therefore, the nonlinear parameter estimation problem at time $t_{k+1}(k=0,1,2, \ldots)$ can be formulated as

$$
\left\{\begin{array}{l}
\boldsymbol{\theta}_{k+1}=\boldsymbol{\theta}_{k}+\boldsymbol{\gamma}_{k} \\
\mathbf{y}_{k+1}=\mathbf{h}_{k+1}\left(\boldsymbol{\theta}_{k+1}, \ddot{\mathbf{u}}_{1: k+1}^{g}\right)+\mathbf{v}_{k+1}
\end{array}\right.
$$

where $\gamma_{k}$ and $\mathbf{v}_{k}$ denote the process noise and measurement noise, respectively, and are assumed to be independent Gaussian white noise processes with zero mean vectors and diagonal covariance matrices $\mathbf{Q}_{k}$ and $\mathbf{R}_{k}$, respectively, i.e., $\gamma_{k} \sim N\left(\mathbf{0}, \mathbf{Q}_{k}\right)$ and $\mathbf{v}_{k} \sim N\left(\mathbf{0}, \mathbf{R}_{k}\right)$. A filtering technique can be used to recursively estimate at least the first two statistical moments of the unknown modeling parameter vector in the nonlinear state-space model given in Equation (4) using the input and measured output response of the system [11].

In this paper, the Extended Kalman filter (EKF), Iterated Extended Kalman filter (IEKF), and Unscented Kalman filter (UKF) are used to recursively estimate the states (i.e., modeling parameters) of the nonlinear statespace model in Equation (4) knowing the input ( $\ddot{\mathbf{u}}_{1: k+1}^{g}$ ) and measured output response of the system $\left(\mathbf{y}_{k+1}\right)$. In the EKF, the nonlinear state-space model is linearized in terms of the modeling parameters $\theta$ (using the DDM), at each time step, about the latest parameter estimates using a first-order Taylor series approximation. The Kalman filter is then applied to the linearized system and the corrected parameter estimates at a given time step are used as the center point for the linearization at the next time step.

In the IEKF, an iterative linearization procedure is performed, at each time step, on the nonlinear measurement equation (second equation in Equation (4)). When linearizing the model at time $t_{k+1}$, the iterative process takes into account the response measurement $\mathbf{y}_{k+1}$ to have a better estimate of $\boldsymbol{\theta}_{k+1}$. The iterative process is terminated when the difference between two consecutive updated parameter estimates (iterations $i-1$ and $i)$ is less than a predefined threshold $\left(\left\|\hat{\boldsymbol{\theta}}_{k+1 \mid k+1}^{i}-\hat{\boldsymbol{\theta}}_{k+1 \mid k+1}^{i-1}\right\| \leq \varepsilon\left\|\hat{\boldsymbol{\theta}}_{k+1 \mid k+1}^{0}\right\|\right)$ or after a maximum number of iterations $\left(N_{\text {iter }}\right)$ is reached. More details about the IEKF can be found in [12]. Figures 2 and 3 summarize the algorithm to update the nonlinear FE model using the EKF and IEKF, respectively.

The UKF utilizes a statistical linear regression approach (i.e., minimizes the sum of the squared errors between the function values of the nonlinear and linearized functions evaluated in the SPs) instead of using analytical linearization (i.e., linearization of the measurement equation using DDM) as in the EKF and IEKF. In the UKF, a set of deterministically chosen sampling points $\left(\vartheta_{i}\right)$, referred to as sigma points (SPs), is selected based on the prior probability density function
(PDF) (mean vector and covariance matrix) of the modeling parameter vector. The sampling points are used to transform the prior probability distribution of the modeling parameters through the nonlinear FE response function (second equation of Equation (4)) and to find an estimate of the probability distribution of the predicted response. To this end, the expected value and covariance matrix of the predicted response are computed from the sample mean and sample covariance matrix of the transformed SPs. The UKF also uses a predictioncorrection scheme to recursively estimate the expected value and covariance matrix of the modeling parameter vector. Figure 4 summarizes the algorithm to update the nonlinear FE model using the UKF.

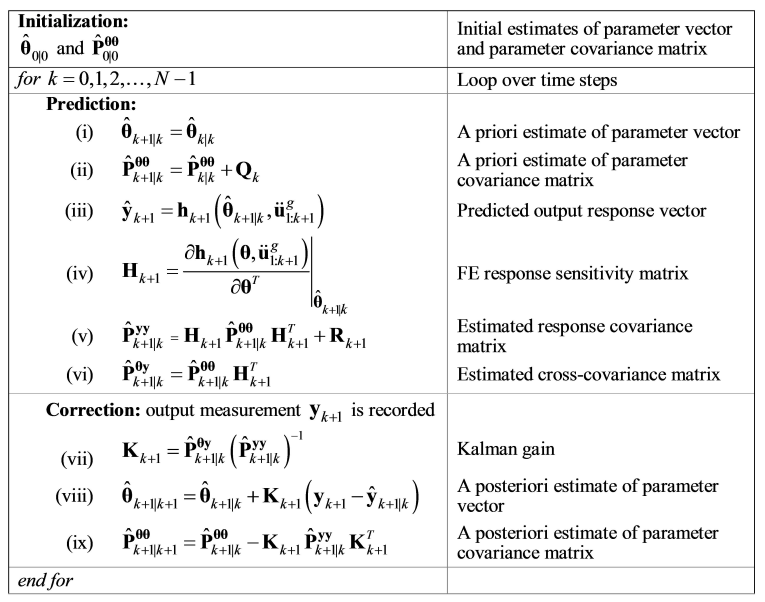

Figure 2. Algorithm for nonlinear FE model updating using the EKF.

\begin{tabular}{|c|c|}
\hline $\begin{array}{l}\text { Initialization: } \\
\hat{\boldsymbol{\theta}}_{0 \mid 0} \text { and } \hat{\mathbf{P}}_{0 \mid 0}^{\theta \theta}\end{array}$ & $\begin{array}{l}\text { Initial estimates of parameter } \\
\text { vector and parameter covariance } \\
\text { matrix }\end{array}$ \\
\hline \multirow{2}{*}{$\begin{array}{l}\text { for } k=0,1,2, \ldots, N-1 \\
\text { Prediction: }\end{array}$} & Loop over time steps \\
\hline & \\
\hline (i) $\hat{\boldsymbol{\theta}}_{k+1 \mid k}=\hat{\boldsymbol{\theta}}_{k \mid k}$ & $\begin{array}{l}\text { A priori estimate of parameter } \\
\text { vector }\end{array}$ \\
\hline (ii) $\hat{\mathbf{P}}_{k+\| l k}^{\theta \theta}=\hat{\mathbf{P}}_{k \mid k}^{\theta \theta}+\mathbf{Q}_{k}$ & $\begin{array}{l}\text { A priori estimate of parameter } \\
\text { covariance matrix }\end{array}$ \\
\hline \multirow{2}{*}{ 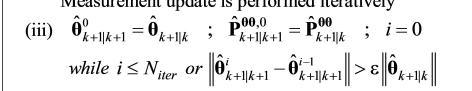 } & $\begin{array}{l}\text { Initialize the iterated EKF } \\
\text { estimate }\end{array}$ \\
\hline & Loop over measurement update \\
\hline & Predicted output response vector \\
\hline (v) $\quad \mathbf{H}_{k+1}^{i}=\left.\frac{\partial \mathbf{h}_{k+1}\left(\boldsymbol{\theta}, \mathbf{u}_{1: k+1}^{g}\right)}{\partial \boldsymbol{\theta}^{T}}\right|_{\hat{\mathbf{\theta}}^{i}}$ & FE response sensitivity matrix \\
\hline \multirow{2}{*}{ 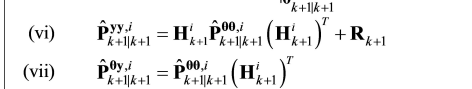 } & $\begin{array}{l}\text { Estimated response covariance } \\
\text { matrix }\end{array}$ \\
\hline & $\begin{array}{l}\text { Estimated cross-covariance } \\
\text { matrix }\end{array}$ \\
\hline \multicolumn{2}{|l|}{ Correction: output measurement $\mathbf{y}_{k+1}$ is recorded } \\
\hline 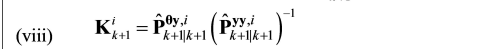 & Kalman gain \\
\hline (ix) $\begin{aligned} & \hat{\boldsymbol{\theta}}_{k+1 \mid k+1}^{i+1}=\hat{\boldsymbol{\theta}}_{k+1 \mid k}+\mathbf{K}_{k+1}^{i}\left[\mathbf{y}_{k+1}-\hat{\mathbf{y}}_{k+1 \mid k+1}^{i}\right. \\
&\left.-\mathbf{H}_{k+1}^{i}\left(\hat{\boldsymbol{\theta}}_{k+1 \mid k}-\hat{\boldsymbol{\theta}}_{k+1 \mid k+1}^{i}\right)\right]\end{aligned}$ & $\begin{array}{l}\text { A posteriori estimate of parameter } \\
\text { vector }\end{array}$ \\
\hline \multirow[t]{2}{*}{ (x) $\quad \hat{\mathbf{P}}_{k+1 \| k+1}^{\theta \theta, i+1}=\hat{\mathbf{P}}_{k+1 \mid k}^{\theta \theta}-\mathbf{K}_{k+1}^{i} \hat{\mathbf{P}}_{k++\mid k+1}^{y, i}\left(\mathbf{K}_{k+1}^{i}\right)^{T}$} & $\begin{array}{l}\text { A posteriori estimate of parameter } \\
\text { covariance matrix }\end{array}$ \\
\hline & \\
\hline \multirow{2}{*}{$\begin{aligned} \text { (xi) } & \hat{\boldsymbol{\theta}}_{k+1 \| k+1}=\hat{\boldsymbol{\theta}}_{k+1 \| k+1, N_{\text {ier }}+1} \\
\text { (xii) } & \hat{\mathbf{P}}_{k+1 \| k+1}^{\theta \theta}=\hat{\mathbf{P}}_{k+1 \mid k+1, N_{\text {ier }}+1}^{\theta 0}\end{aligned}$} & $\begin{array}{l}\text { Final a posteriori estimate of } \\
\text { parameter vector }\end{array}$ \\
\hline & $\begin{array}{l}\text { Final a posteriori estimate of } \\
\text { parameter vector }\end{array}$ \\
\hline
\end{tabular}

Figure 3. Algorithm for nonlinear FE model updating using the IEKF. 


\begin{tabular}{|c|c|}
\hline $\begin{array}{l}\text { Initialization: } \\
\hat{\boldsymbol{\theta}}_{0 \mid 0} \text { and } \hat{\mathbf{P}}_{000}^{\theta \theta}\end{array}$ & $\begin{array}{l}\text { Initial estimates of parameter vector } \\
\text { and parameter covariance matrix }\end{array}$ \\
\hline for $k=0,1,2, \ldots$ & Loop over time steps \\
\hline Prediction: & \\
\hline (i) $\boldsymbol{\vartheta}_{k+1 \mid k}^{(i)}=\boldsymbol{\vartheta}_{k \mid k}^{(i)}$ & Generate SPs \\
\hline (ii) $\quad \hat{\boldsymbol{\theta}}_{k+\| k}=\hat{\boldsymbol{\theta}}_{k \mid k}$ & $\begin{array}{l}\text { A priori estimate of parameter } \\
\text { vector }\end{array}$ \\
\hline (iii) $\hat{\mathbf{P}}_{k+1 \mid k}^{\theta \theta}=\hat{\mathbf{P}}_{k \mid k}^{\theta \theta}+\mathbf{Q}_{k}$ & $\begin{array}{l}\text { A priori estimate of parameter } \\
\text { covariance matrix }\end{array}$ \\
\hline (iv) $\quad \boldsymbol{y}_{k+1}^{(i)}=\mathbf{h}_{k+1}\left(\boldsymbol{\vartheta}_{k+1 \mid k}^{(i)}, \mathbf{u}_{1: k+1}^{g}\right)$ & Output response vector for each SP \\
\hline (v) $\quad \hat{\mathbf{y}}_{k+\| k}=\sum_{i=1}^{2 n_{0}+1} W_{m}^{(i)} y_{k+1}^{(i)}$ & Predicted output response vector \\
\hline (vi) $\quad \hat{\mathbf{P}}_{k+1 \mid k}^{y \boldsymbol{y}}=\sum_{i=1}^{2 n_{+1}+1} W_{c}^{(i)}\left[\boldsymbol{y}_{k+1}^{(i)}-\hat{\mathbf{y}}_{k+1 \| k}\right]\left[\boldsymbol{y}_{k+1}^{(i)}-\hat{\mathbf{y}}_{k+1 \mid k}\right]^{T}+\mathbf{R}_{k+1}$ & $\begin{array}{l}\text { Estimated response covariance } \\
\text { matrix }\end{array}$ \\
\hline 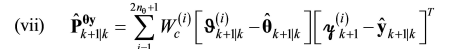 & Estimated cross-covariance matrix \\
\hline Correction: output measurement $\mathbf{y}_{k+1}$ is recorded & \\
\hline (viii) $\quad \mathbf{K}_{k+1}=\hat{\mathbf{P}}_{k+1 \mid k}^{\mathbf{a y}}\left(\hat{\mathbf{P}}_{k+1 \mid k}^{\mathbf{y y}}\right)^{-1}$ & Kalman gain \\
\hline (ix) $\hat{\boldsymbol{\theta}}_{k+1 \mid k+1}=\hat{\boldsymbol{\theta}}_{k+1 \| k}+\mathbf{K}_{k+1}\left(\mathbf{y}_{k+1}-\hat{\mathbf{y}}_{k+1 \| k}\right)$ & $\begin{array}{l}\text { A posteriori estimate of parameter } \\
\text { vector }\end{array}$ \\
\hline (x) $\quad \hat{\mathbf{P}}_{k+1 \| k+1}^{\boldsymbol{\theta \theta}}=\hat{\mathbf{P}}_{k+1 \| k}^{\boldsymbol{\theta \theta}}-\mathbf{K}_{k+1} \hat{\mathbf{P}}_{k+1 \| k}^{\mathbf{y y}} \mathbf{K}_{k+1}^{T}$ & $\begin{array}{l}\text { A posteriori estimate of parameter } \\
\text { covariance matrix }\end{array}$ \\
\hline
\end{tabular}

Figure 4. Algorithm for nonlinear FE model updating using the UKF.

More information on the nonlinear FE model updating using the EKF and UKF can be found in [8] and [7], respectively. It is noted that in Figures 2, 3, and 4 $\hat{\mathbf{P}}_{k+1 \mid k}^{\mathbf{x z}}=E\left[\left(\mathbf{x}_{k+1}-\hat{\mathbf{x}}_{k+1 \mid k}\right)\left(\mathbf{z}_{k+1}-\hat{\mathbf{z}}_{k+1 \mid k}\right)^{T} \mid \mathbf{y}_{1}, \mathbf{y}_{2}, \ldots \mathbf{y}_{k}\right] \quad$ and $\hat{\mathbf{x}}_{k+1 \mid k}=E\left[\mathbf{x}_{k+1} \mid \mathbf{y}_{1}, \mathbf{y}_{2}, \ldots, \mathbf{y}_{k}\right]$.

\section{Verification example}

A three-dimensional five-story two-by-one bay RC frame building subjected to bidirectional horizontal seismic excitation is considered to verify the proposed FE model updating methodology using the EKF, IEKF, and UKF and compare their performance. A mechanics-based nonlinear FE model of the building, developed in the open-source object-oriented software framework OpenSees [13], is used to simulate the response of the building. In the estimation phase, the simulated response data are contaminated by additive white Gaussian noise (AWGN) and used as measured output (y) to estimate the parameters characterizing the nonlinear material constitutive laws of the concrete and reinforcing steel.

\subsection{Nonlinear finite element model description and input earthquake motions}

The structure is designed as an intermediate momentresisting RC frame located in downtown Seattle, WA, with Site Class D soil conditions, a short-period spectral acceleration $S_{M S}=1.37 g$, and a one-second spectral acceleration $S_{M I}=0.53 g$. The building has two bays in the longitudinal (X) direction and one bay in the transverse (Z) direction, with plan dimensions of $10.0 \times 6.0 \mathrm{~m}$, respectively. The frame has 5 stories with a floor-to-floor height of $4.0 \mathrm{~m}$. Masses as well as dead and live loads are computed according to the 2006 International Building Code [14].

The building has six identical $0.45 \times 0.45 \mathrm{~m} \mathrm{RC}$ columns reinforced with 8 \#8 longitudinal reinforcement bars and \#3@150 mm as transverse reinforcement. Grade 75 reinforcing steel is used for the columns.
Longitudinal beams have a square cross-section of $0.40 \times 0.40 \mathrm{~m}$ and are reinforced with $6 \# 8$ longitudinal reinforcement bars and \#3@100 mm as transverse reinforcement. Transverse beams have a rectangular cross-section of $0.40 \times 0.45 \mathrm{~m}$ and are reinforced with 8 \#8 longitudinal reinforcement bars and \#3 @ $100 \mathrm{~mm}$ as transverse reinforcement. Grade 60 reinforcing steel is used for the beams in both directions. Figure 5 shows the overall geometry of the building and the cross-sections of beams and columns.

Translational components of ground acceleration recorded at the Sylmar County Hospital during the 1994 Northridge earthquake are used as input base excitation (Figure 6). Components $360^{\circ}$ and $90^{\circ}$ are applied in the longitudinal and transverse direction of the building, respectively. The earthquake ground motions were recorded at a sampling rate of $50 \mathrm{~Hz}$, filtered through a band-pass filter with cutoff frequencies of 0.1 and 23.0 $\mathrm{Hz}$, and have $N=550$ data samples. The peak ground acceleration (PGA) of components $360^{\circ}$ and $90^{\circ}$ were $0.84 g$ and $0.60 g$, respectively.
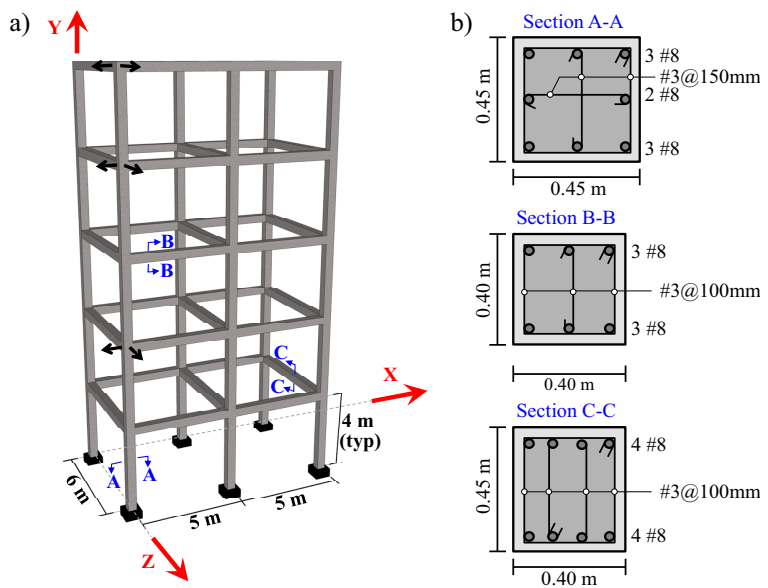

Figure 5. RC frame building: (a) Isometric view, (b) Crosssection of beams and columns.

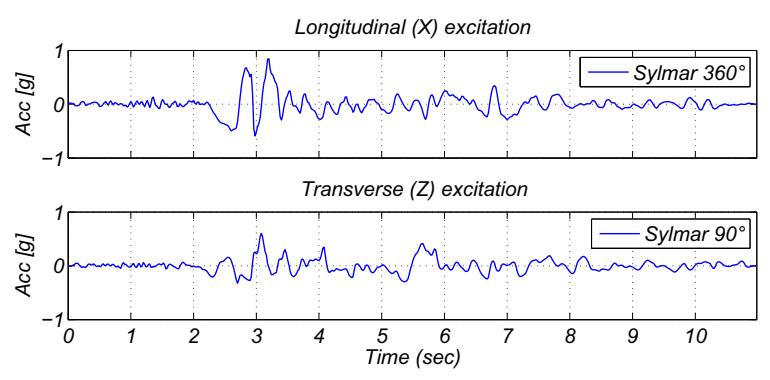

Figure 6. Ground acceleration records used as seismic input motions.

The modified Giuffré-Menegotto-Pinto model [15] is used to model the nonlinear uniaxial stress-strain behavior of the longitudinal steel reinforcing bars. This material model is governed by eight parameters, three of which are primary physical parameters, while the other five are secondary parameters controlling the curvature of the hysteresis branches between consecutive strain reversal points. The physical material parameters consist 
of the elastic modulus $\left(E_{s}\right)$, initial yield strength $\left(f_{y}\right)$, and strain hardening ratio $(b)$; they are considered unknown parameters in the estimation phase. The Popovics-Saenz model [16-18] is used to model the concrete fibers. Five material parameters define this uniaxial stress-strain material model. These parameters are the modulus of elasticity $\left(E_{c}\right)$, peak compressive strength $\left(f_{c}\right)$, strain at peak compressive strength $\left(\varepsilon_{\mathrm{c}}\right)$, crushing strength $\left(f_{u}\right)$, and strain at crushing strength $\left(\varepsilon_{\mathrm{u}}\right)$. The values of $f_{c}, \varepsilon_{\mathrm{c}}, f_{u}$, and $\varepsilon_{\mathrm{u}}$ correspond to the confined state of concrete and are determined based on the initial properties of the concrete material. Parameters $f_{c}$ and $\varepsilon_{\mathrm{c}}$ account for the confinement effects of the transverse reinforcement according to Mander et al. [19], whereas $\varepsilon_{\mathrm{u}}$ is obtained as suggested by Scott et al. [20].

Figure 7 shows the uniaxial material models used for the concrete and reinforcing steel fibers together with their assumed to be unknown parameters. A set of material parameter values, referred to as true values hereafter, are assumed for the concrete and reinforcing steel materials in order to simulate the response of the structure. There true material parameter values are: $E_{s-\text { col }}^{\text {true }}=200 \mathrm{GPa}, \quad f_{y-\text { col }}^{\text {true }}=517 \mathrm{MPa}, \quad b_{\text {col }}^{\text {true }}=0.01$, $E_{s-\text { beam }}^{\text {true }}=200 \mathrm{GPa}, f_{y \text {-beam }}^{\text {true }}=414 \mathrm{MPa}, b_{\text {beam }}^{\text {true }}=0.05$, $E_{c}^{\text {true }}=27600 \mathrm{MPa}, \quad f_{c}^{\text {true }}=40 \mathrm{MPa}, \quad$ and $\varepsilon_{c}^{\text {true }}=0.0035$. It is noteworthy that $f_{u}$ and $\varepsilon_{\mathrm{u}}$ are not considered as parameters to be estimated, since they have negligible effects on the (simulated) recorded response of the structure in this case study.
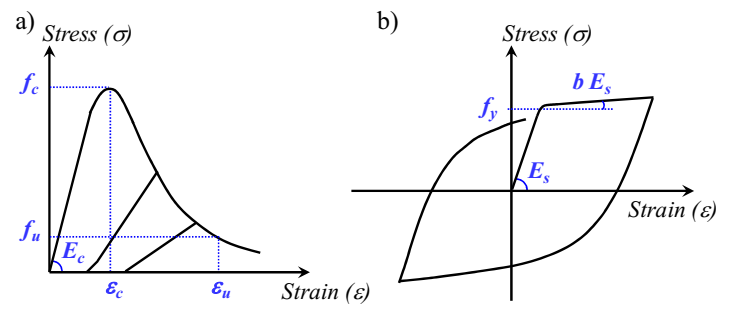

Figure 7. Uniaxial material models used in the FE model: (a) Concrete, (b) Reinforcing steel.

\subsection{Estimation results}

Nine modeling parameters characterizing the concrete and reinforcing steel constitutive laws are considered as unknown and define the model parameter vector to be estimated, i.e., $\boldsymbol{\theta}=\left[E_{s-c o l}, f_{y \text {-col }}, b_{\text {col }}, E_{\text {s-beam }}, f_{\text {y-beam }}, b_{\text {beam }}\right.$, $\left.E_{c}, f_{c}, \varepsilon_{c}\right]^{\mathrm{T}} \in \mathbb{R}^{9 \times 1}$. The response of the structure is simulated using the true values of the parameters (see Section 4.1) and is referred to as true response hereafter. After completion of the response simulation, the true relative horizontal acceleration responses at the $3^{\text {rd }}$ floor, $5^{\text {th }}$ floor, and roof levels in both the longitudinal (X) and transverse $(\mathrm{Z})$ directions (see black arrows in Figure 5) are polluted by AWGN, representing output measurement noise, and used to define the measured response data $(\mathbf{y})$. The measured response data together with the two horizontal translational components of ground acceleration $\left(\ddot{\mathbf{u}}^{g}\right)$ recorded at the Sylmar station during the 1994 Northridge earthquake (Figure 6) are used to estimate the model parameter vector and to update the nonlinear FE model of the structure. It is noted that the same FE model of the structure is used for response simulation and parameter estimation, i.e., the effects of modeling uncertainty is not considered here.

A $1.0 \% \mathrm{~g}$ root-mean-square (RMS) zero-mean white Gaussian output measurement noise is added to each simulated relative acceleration response time history, i.e., the actual covariance matrix of the output measurement noise vector is $(0.01 \times 9.81)^{2}=0.96 \times 10^{-2} \mathbf{I}_{6}\left(\mathrm{~m} / \mathrm{s}^{2}\right)^{2}$ where $\mathbf{I}_{i}=i \times i$ identity matrix. Statistically independent realizations of output measurement noise are considered for different acceleration responses. In the estimation phase, it is assumed that the output measurement noise is a zero-mean white Gaussian process with a covariance matrix $\mathbf{R}_{k+1}=\mathbf{R}=0.47 \times 10^{-2} \mathbf{I}_{6}\left(\mathrm{~m} / \mathrm{s}^{2}\right)^{2}$, i.e., a standard deviation (or RMS) of $0.7 \% \mathrm{~g}$ is estimated for the output measurement noise. The assumed amplitude of the measurement noise is purposely taken different from the (true) amplitude, since in practice the measurement noise amplitude is unknown. The amplitude of the output measurement noise can however be estimated based on the characteristics of the sensors and DAQ system, experience, and engineering judgment. Time-invariant first- and second-order statistics are assumed for the process noise $\gamma_{k}$, with zero-mean and covariance matrix $\mathbf{Q}_{k}=\mathbf{Q}$. The diagonal entries of $\mathbf{Q}$ are assumed equal to $\left(q \times \hat{\theta}_{0 \mid 0}^{i}\right)^{2}$ where $i=1, \ldots, 9$ and $q=1 \times 10^{-5}$, i.e., the RMS of the process noise is assumed equal to $1 \times 10^{-5}$ the initial prior mean estimate of the material parameters $\left(\hat{\boldsymbol{\theta}}_{0 \mid 0}\right)$. For the filter initialization, $\hat{\boldsymbol{\theta}}_{0 \mid 0} / \boldsymbol{\theta}^{\text {true }}=[0.70$, $1.30,1.25,1.30,0.80,0.75,1.20,0.85,0.90]$ is assumed as the initial guess of the expected value of the modeling parameters. The initial estimate of the covariance matrix of the modeling parameters, $\hat{\mathbf{P}}_{0 \mid 0}^{\theta \theta}$, is assumed to be diagonal (i.e., initial estimates of the modeling parameters are assumed statistically uncorrelated). Diagonal entries of $\hat{\mathbf{P}}_{0 \mid 0}^{\boldsymbol{\theta \theta}}$ are taken as $\left(p \times \hat{\theta}_{0 \mid 0}^{i}\right)^{2}$ where $i=1, \ldots, 9$ and $p=0.15$, i.e., assuming a coefficient of variation of $15 \%$ for the initial prior Bayesian estimate of the parameters.

The algorithms summarized in Figures 2, 3, and 4 are used to recursively estimate the expected value and covariance matrix of the modeling parameters at each time step. Figure 8 shows the time histories of the a posteriori estimates of the material model parameters while Table 1 reports the final a posteriori estimates of these parameters, $\hat{\boldsymbol{\theta}}_{N \mid N}$, (which correspond to stable and converged values) normalized by their corresponding true values.

It is observed that all material model parameters are accurately estimated with relative errors less than $5 \%$. The EKF, IEKF, and UKF provide accurate estimates of the modeling parameters; however, the convergence of the UKF is smoother and faster than that of the EKF and IEKF. 

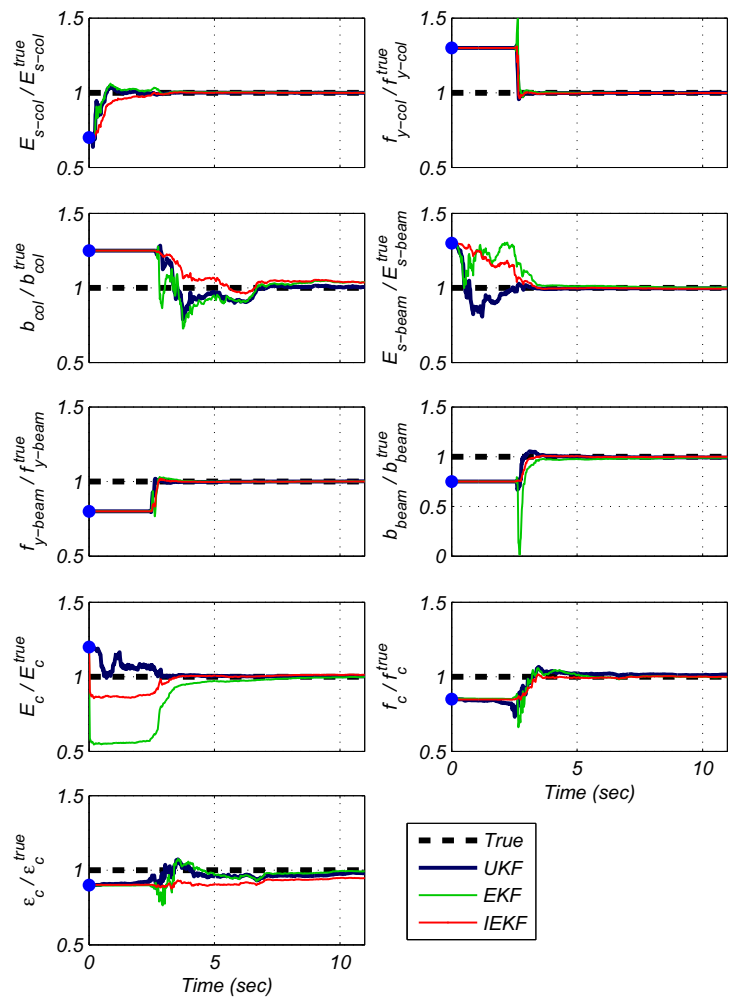

Figure 8. Time histories of the a posteriori estimates of the material model parameters.

Table 1. Final estimate and coefficient of variation (in parentheses) of the modeling parameters.

\begin{tabular}{|c|c|c|c|c|c|c|c|c|c|}
\hline \multirow{3}{*}{ Filter } & \multicolumn{9}{|c|}{ Modeling parameter } \\
\hline & $E_{s-c o l}$ & $f_{y-c o l}$ & $b_{c o l}$ & $E_{s-\text { beam }}$ & $f_{y \text {-beam }}$ & $b_{\text {beam }}$ & $E_{c}$ & $f_{c}$ & $\varepsilon_{c}$ \\
\hline & $\overline{E_{s-c o l}^{\text {true }}}$ & $\overline{f_{y-c o l}^{\text {true }}}$ & $\overline{b_{\text {col }}^{\text {true }}}$ & $\overline{E_{\text {s-beam }}^{\text {true }}}$ & $\overline{f_{y \text {-beam }}^{\text {true }}}$ & $\overline{b_{\text {beam }}^{\text {true }}}$ & $\overline{E_{c}^{\text {true }}}$ & $\overline{f_{c}^{\text {true }}}$ & $\overline{\varepsilon_{c}^{\text {true }}}$ \\
\hline UKF & $\begin{array}{c}1.00 \\
(0.09)\end{array}$ & $\begin{array}{c}1.00 \\
(0.06)\end{array}$ & $\begin{array}{c}1.01 \\
(1.47)\end{array}$ & $\begin{array}{c}1.00 \\
(0.13)\end{array}$ & $\begin{array}{c}1.00 \\
(0.08)\end{array}$ & $\begin{array}{c}0.99 \\
(0.32)\end{array}$ & $\begin{array}{c}1.00 \\
(0.21)\end{array}$ & $\begin{array}{c}1.01 \\
(0.87)\end{array}$ & \begin{tabular}{|c|}
0.98 \\
$(1.88)$
\end{tabular} \\
\hline EKF & $\begin{array}{c}1.00 \\
(0.11)\end{array}$ & $\begin{array}{c}1.00 \\
(0.07)\end{array}$ & $\begin{array}{c}1.04 \\
(1.58)\end{array}$ & $\begin{array}{c}1.00 \\
(0.15)\end{array}$ & $\begin{array}{c}1.00 \\
(0.09)\end{array}$ & $\begin{array}{c}0.99 \\
(0.31)\end{array}$ & $\begin{array}{c}1.00 \\
(0.33)\end{array}$ & $\begin{array}{c}1.00 \\
(0.95)\end{array}$ & $\begin{array}{c}0.99 \\
(2.11) \\
\end{array}$ \\
\hline IEKF & $\begin{array}{c}1.00 \\
(0.11)\end{array}$ & $\begin{array}{c}1.00 \\
(0.07)\end{array}$ & $\begin{array}{c}1.04 \\
(1.51)\end{array}$ & $\begin{array}{c}1.00 \\
(0.15)\end{array}$ & $\begin{array}{c}1.00 \\
(0.10)\end{array}$ & $\begin{array}{c}1.00 \\
(0.31)\end{array}$ & $\begin{array}{c}1.01 \\
(0.35) \\
\end{array}$ & \begin{tabular}{|c|}
1.00 \\
$(0.82)$
\end{tabular} & $\begin{array}{c}0.95 \\
(1.71) \\
\end{array}$ \\
\hline
\end{tabular}

The relative RMS error (RRMSE) between the true responses and their counterparts obtained using the final estimates of the modeling parameters $\left(\hat{\boldsymbol{\theta}}_{N \mid N}\right)$ are computed and reported in Table 2. The RRMS error between two signals $\mathbf{s}^{1}$ and $\mathbf{s}^{2}$ with $\mathbf{s}^{1}$ taken as the reference signal is computed as $\operatorname{RRMSE}[\%]=\sqrt{\left[1 / N s \sum_{k=1}^{N s}\left(s_{k}^{1}-s_{k}^{2}\right)^{2}\right]} / \sqrt{\left[1 / N s \sum_{k=1}^{N s}\left(s_{k}^{1}\right)^{2}\right]} \times 100$, where $N_{S}$ denotes the total number of data samples. In Table 2, output response $\mathrm{a}_{i j}$ corresponds to the relative acceleration time history at story $i$ in direction $j$, where $l$ and $t$ stand for the longitudinal and transverse directions, respectively. The RRMSEs between the true responses and their counterparts obtained using the initial estimate of the modeling parameters $\left(\hat{\boldsymbol{\theta}}_{0 \mid 0}\right)$ are also included for comparison purposes. Comparing the RRMSEs between the true responses and their estimated counterparts based on the initial and final estimates of the material model parameters illustrates the successful performance of the proposed FE model updating framework using the EKF, IEKF, and UKF. The discrepancies between the true responses and the corresponding responses of the updated FE model are almost negligible with RRMSEs below $1.0 \%$. The low RRMSEs obtained, in spite of estimates of material parameters $b_{c o l}$ and $\varepsilon_{\mathrm{c}}$ not fully converged to their true values (see Table 1), also confirm that the measured acceleration responses do not contain enough information about modeling parameters $b_{c o l}$ and $\varepsilon_{\mathrm{c}}$ for their accurate estimation. It is observed that the UKF outperforms the EKF and IEKF, because it yields slightly lower RRMSEs for all the measured responses.

Table 2.Relative RMS error between true and estimated (using $\hat{\boldsymbol{\theta}}_{0 \mid 0}$ and $\hat{\boldsymbol{\theta}}_{N \mid N}$ ) relative acceleration response time histories.

\begin{tabular}{|c|c|c|c|c|c|c|c|}
\cline { 3 - 8 } \multicolumn{1}{c|}{} & \multirow{2}{*}{ Filter } & \multicolumn{6}{|c|}{ Output response measurement $(\mathbf{y})$} \\
\cline { 3 - 8 } \multicolumn{1}{c|}{} & $\mathrm{a}_{3 l}$ & $\mathrm{a}_{5 l}$ & $\mathrm{a}_{6 l}$ & $\mathrm{a}_{3 t}$ & $\mathrm{a}_{5 t}$ & $\mathrm{a}_{6 t}$ \\
\hline$\hat{\boldsymbol{\theta}}_{0 \mid 0}$ & - & 58.89 & 38.33 & 37.86 & 39.62 & 45.48 & 40.64 \\
\hline \multirow{2}{*}{$\hat{\boldsymbol{\theta}}_{N \mid N}$} & UKF & 0.41 & 0.28 & 0.23 & 0.24 & 0.24 & 0.25 \\
\cline { 2 - 8 } & EKF & 0.56 & 0.28 & 0.22 & 0.33 & 0.25 & 0.30 \\
\cline { 2 - 8 } & IEKF & 0.47 & 0.30 & 0.29 & 0.49 & 0.45 & 0.51 \\
\hline
\end{tabular}

\section{Conclusions}

This paper studied and compared the performance of a new framework to update mechanics-based nonlinear structural finite element (FE) models when different variants of the Kalman filter for nonlinear state-space models are used as estimation tool. The framework uses recorded input-output data to estimate unknown parameters of advanced mechanics-based nonlinear FE models of the structure of interest, using the Extended Kalman filter (EKF), iterated Extended Kalman filter (IEKF), and Unscented Kalman filter (UKF). The updated FE model provides accurate and complete information about potential damage in the structure, including loss of stiffness, strength degradation, loss of ductility capacity and history of inelastic deformations at the global and local levels.

The proposed methodology was verified using numerically simulated response data for a realistic threedimensional five-story two-by-one bay reinforced concrete (RC) frame building subjected to bi-directional horizontal earthquake excitation. Parameters characterizing the nonlinear material constitutive laws of the reinforcing steel and concrete materials were successfully estimated using the seismic input data together with limited response data (6 acceleration response time histories). Excellent results were obtained with the three Bayesian filters, but the UKF outperformed the EKF and IEKF when the relative root-mean-square errors between the true and simulated (based on the final estimate of the modelling parameters) relative acceleration responses were compared. In addition, the results suggest that convergence of the estimation of modeling parameters is smoother and faster when the UKF is utilized.

It is noteworthy that the proposed framework is not limited to certain types of FE models, loading conditions, 
or FE model parameters. Different types of material constitutive models (e.g., plasticity, smeared crack), various FEs (e.g., beam-column, shell, solid elements), and different types of analysis (e.g., quasi-static, timedependent, dynamic) can be used with the proposed framework. Furthermore, other time-invariant FE modeling parameters (e.g., inertia, damping, geometric, and constraint parameters) can also be incorporated in the estimation procedure.

\section{Acknowledgements}

Partial support of this research by the UCSD Academic Senate under Research Grant RN091G-CONTE is gratefully acknowledged. Any opinions, findings, and conclusions or recommendations expressed in this paper are those of the authors and do not necessarily reflect those of the sponsor.

\section{References}

1. M.I. Friswell, J.E. Mottershead. Finite element model updating in structural dynamics. (Kluwer Academic Publishers, Dordrecht, The Netherlands, 1995).

2. E. Simoen, G. De Roeck, G. Lombaert. "Dealing with uncertainty in model updating for damage assessment: A review." Mechanical Systems and Signal Processing 56-57, 123-149 (2015).

3. J. Ching, J.L. Beck, K.A. Porter, R. Shaikhutdinov. "Bayesian state estimation method for nonlinear systems and its application to recorded seismic response." ASCE Journal of Engineering Mechanics 132(4), 396-410 (2006).

4. W. Song, S.J. Dyke. "Real-time dynamic model updating of a hysteretic structural system." ASCE Journal of Structural Engineering 140(3), 04013082 (2014).

5. J. Yang, Y. Xia, C.H. Loh. "Damage Detection of Hysteretic Structures with Pinching Effect." ASCE Journal of Engineering Mechanics 140(3), 462-472 (2014).

6. S. Shahidi, S. Pakzad. "Generalized response surface model updating using time domain data." ASCE Journal of Structural Engineering 140, SPECIAL ISSUE: Computational Simulation in Structural Engineering, A4014001 (2014).

7. R. Astroza, E. Ebrahimian, J.P. Conte. "Material parameter identification in distributed plasticity FE models of frame-type structures using nonlinear stochastic filtering." ASCE Journal of Engineering Mechanics 141(5), 04014149 (2015).

8. H. Ebrahimian, R. Astroza, J.P. Conte. "Extended Kalman filter for material parameter estimation in nonlinear structural finite element models using direct differentiation method." Earthquake Engineering \& Structural Dynamics, In press (2015).

9. G.G. Deierlein, A.M. Reinhorn, M.R. Willford. Nonlinear structural analysis for seismic design: A guide for practicing engineers. NIST GCR 10-917-5, National Institute of Standards and Technology, Gaithersburg, MD (2010).
10. F.F. Taucer, E. Spacone, E., F.C. Filippou. A fiber beam-column element for seismic response analysis of reinforced concrete structures. Report 91/17, EERC, Earthquake Engineering Research Center (EERC), University of California, Berkeley (1991).

11. A.J. Haug. A tutorial on Bayesian estimation and tracking techniques applicable to nonlinear and nonGaussian processes. Technical Rep. MTR 05W0000004, MITRE, McLean, VA (2005).

12. D. Simon. Optimal State Estimation: Kalman, $H$ Infinity, and Nonlinear Approaches. Hoboken, New Jersey, John Wiley \& Sons, Inc (2006).

13. OpenSees. Open System for Earthquake Engineering Simulation. http://opensees.berkeley.edu/. [Accessed September 2013].

14. International Code Council (ICC), International building code, 2006 ed., Falls Church, VA (2006).

15. F.C. Filippou, E.P. Popov, V.V. Bertero. Effects of bond deterioration on hysteretic behavior of reinforced concrete joints. UCB/EERC-83/19, Berkeley, CA: EERC Report 83-19, Earthquake Engineering Research Center (1983).

16. S. Popovics. "A numerical approach to the complete stress-strain curve of concrete." Cement Concrete Research 3(5), 583-599 (1973).

17. I.P. Saenz. "Discussion of 'Equation for the stressstrain curve of concrete, by P. Desay and S. Krishan'." American Concrete Institute (ACI) Journal 61(9), 1229-1235 (1964).

18. Balan, T.A., Spacone, E., and Kwon, M., "A 3D hypoplastic model for cyclic analysis of concrete structures." Engineering Structures 23(4), 333-342. doi:10.1016/S0141-0296(00)00048-1 (2001).

19. J.B.Mander, M.J.N. Priestley, R. Park. "Theoretical stress-strain model for confined concrete." ASCE Journal of Structural Engineering 114(8), 18041826 (1988).

20. B.D. Scott, R. Park, M.J.N. Priestley. "Stress-strain behavior of concrete confined by overlapping hoops at low and high strain rates." American Concrete Institute (ACI) Journal 79(1), 13-27 (1982). 